\title{
PRUNED DISCRETE RANDOM SAMPLES
}

\author{
RUDOLF GRÜBEL, ${ }^{*}$ Leibniz Universität Hannover \\ PAWEŁ HITCZENKO,** Drexel University
}

\begin{abstract}
Let $X_{i}, i \in \mathbb{N}$, be independent and identically distributed random variables with values in $\mathbb{N}_{0}$. We transform ('prune') the sequence $\left\{X_{1}, \ldots, X_{n}\right\}, n \in \mathbb{N}$, of discrete random samples into a sequence $\left\{0,1,2, \ldots, Y_{n}\right\}, n \in \mathbb{N}$, of contiguous random sets by replacing $X_{n+1}$ with $Y_{n}+1$ if $X_{n+1}>Y_{n}$. We consider the asymptotic behaviour of $Y_{n}$ as $n \rightarrow \infty$. Applications include path growth in digital search trees and the number of tables in Pitman's Chinese restaurant process if the latter is conditioned on its limit value.
\end{abstract}

Keywords: Chinese restaurant process; digital search trees; geometric distribution; maxima; tail behaviour

2010 Mathematics Subject Classification: Primary 60C05

Secondary 60F99; 60J10

\section{Introduction}

Let $X_{i}, i \in \mathbb{N}$, be independent and identically distributed random variables with values in the set $\mathbb{N}_{0}=\{0,1,2, \ldots\}$ of nonnegative integers. We assume that

$$
p_{k}:=\mathbb{P}\left(X_{i}=k\right)>0 \text { for all } k \in \mathbb{N}_{0} .
$$

In [7] we investigated the gaps in the set $\left\{X_{1}, \ldots, X_{n}\right\} \subset \mathbb{N}_{0}$ and found 'phase transitions' - in the sense of a qualitative change of behaviour - at two standard discrete distributions. Roughly, if the tails $\left(q_{k}\right)_{k \in \mathbb{N}_{0}}$,

$$
q_{k}:=\mathbb{P}\left(X_{i} \geq k\right)=\sum_{j=k}^{\infty} p_{j},
$$

of the underlying distribution are thinner than that of a Poisson distribution then, with probability 1 , the gaps will eventually vanish as $n \rightarrow \infty$. In the range between Poissonian and geometric tail behaviour we still see that the probability of a gap tends to 0 . Finally, if the tails are heavier than that of a geometric distribution then the length of the longest gap in the sample tends to $\infty$ in probability.

In the present paper we consider the 'pruned' samples, by which we mean the sequence of random sets $\left\{0, \ldots, Y_{n}\right\}$ defined inductively by $Y_{1}:=0$ and

$$
Y_{n+1}:=\left\{\begin{array}{ll}
Y_{n}+1 & \text { if } X_{n+1}>Y_{n}, \\
Y_{n} & \text { otherwise, }
\end{array} \quad \text { for all } n \in \mathbb{N} .\right.
$$

Received 1 June 2012; revision received 12 September 2012.

* Postal address: Institut für Mathematische Stochastik, Leibniz Universität Hannover, Postfach 6009, D-30060 Hannover, Germany. Email address: rgrubel@stochastik.uni-hannover.de

** Postal address: Departments of Mathematics and Computer Science, Drexel University, 3141 Chestnut Street, Philadelphia PA 19104, USA. Email address: phitczen@math.drexel.edu

Partially supported by a grant from Simons Foundation (grant no. 208766). 
Hence, values beyond the current range are reduced so that the range remains contiguous. Clearly, this leads to a Markov chain $\left(Y_{n}\right)_{n \in \mathbb{N}}$ with state space $\mathbb{N}_{0}$, starting at 0 . This chain is of pure birth type and thus fully described by the transition probabilities $p_{i, i+1}$ of moving from $i$ to $i+1$. Indeed, here we have $p_{i, i+1}=q_{i+1}$, and it is easy to see that, whenever $i \rightarrow p_{i, i+1}$ decreases and has limit 0 , then the birth chain can be represented by a pruned discrete random sample. Our results can therefore be used to obtain the asymptotic behaviour of a wide class of pure birth processes.

For later use, and also in view of another interpretation of the $Y$-sequence, we need both the 'number of failures' and the 'waiting for the first success' versions of the geometric distribution, writing $Z \sim \mathrm{Geo}_{0}(p)$ if $\mathbb{P}(Z=k)=q^{k} p$ for all $k \in \mathbb{N}_{0}$ and $Z \sim \mathrm{Geo}_{1}(p)$ if $\mathbb{P}(Z=k)=$ $q^{k-1} p$ for all $k \in \mathbb{N}, q=1-p$. We may then regard $\left(Y_{n}\right)_{n \in \mathbb{N}}$ as the (nonhomogeneous) renewal process associated with independent lifetimes $G_{k}, G_{k} \sim \mathrm{Geo}_{1}\left(q_{k}\right)$ for all $k \in \mathbb{N}$.

As in [7], our motivation for the study of these processes comes from the analysis of algorithms and random discrete structures.

Example 1. In the digital search tree (DST) algorithm infinite zero-one sequences $x_{n}=$ $\left(x_{n, i}\right)_{i \in \mathbb{N}} \in\{0,1\}^{\mathbb{N}}, n \in \mathbb{N}$, are stored sequentially into the nodes $u \in \bigcup_{k=0}^{\infty}\{0,1\}^{k}$ of a binary tree in a 'trickle-down' manner: starting at the root we use the digits (entries) $x_{n, i}$ of $x_{n}$ to choose either the left (if $x_{n, i}=0$ ) or right (if $x_{n, i}=1$ ) successor of an occupied node until an empty node is found. This algorithm and its properties are discussed in [4], [9], and [10].

Suppose now that the $x_{n} \mathrm{~s}$ are independent and that they all have the same distribution $\mu$. For any node $u=\left(u_{1}, \ldots, u_{k}\right)$, let $\mu_{u}$ be the probability that $x_{n}$ has prefix $u$, i.e. that $x_{n, i}=u_{i}$ for $i=1, \ldots, k$. Along a specified path $v=\left(v_{i}\right)_{i \in \mathbb{N}} \in\{0,1\}^{\infty}$ the sequence of random trees then grows like a pruned discrete random sample, with $q_{k}$ equal to $\mu_{u}, u:=\left(v_{1}, \ldots, v_{k}\right)$. Important special cases are the symmetric binomial model, with $\mu_{u}=2^{-|u|},|u|$ being the depth of the node $u$, and the general binomial model with parameter $\theta \in(0,1)$, where $\mu_{u}=\theta^{l}(1-\theta)^{m}$, $l$ and $m$ being the number of entries of $u$ that are equal to 0 and 1, respectively. More general $\mu$ s appear in connection with the binary search tree (BST) algorithm if we condition on the value of the limit tree; see [5].

Example 2. The following construction of a sequence $\left(X_{n}\right)_{n \in \mathbb{N}}$ of random partitions

$$
X_{n}=\left\{B_{n, 1}, \ldots, B_{n, k_{n}}\right\}
$$

of the sets $\{1, \ldots, n\}, n \in \mathbb{N}$, is known as Pitman's Chinese restaurant process; see, e.g. [11]. Starting with $X_{1}=\{\{1\}\}$ we obtain $X_{n+1}$ from $X_{n}$ by choosing the next partition, now of $\{1, \ldots, n, n+1\}$, to be

$$
X_{n+1}=\left\{B_{n, 1}, \ldots, B_{n, k_{n}},\{n+1\}\right\}
$$

with probability $1 /(n+1)$ and

$$
X_{n+1}=\left\{B_{n, 1}, \ldots, B_{n, j-1}, B_{n, j} \cup\{n+1\}, \ldots, B_{n, k_{n}}\right\}
$$

with probability \# $B_{n, j} /(n+1), j=1, \ldots, k_{n}$. Here \#A denotes the number of elements of the set $A$. Clearly, $\left(X_{n}\right)_{n \in \mathbb{N}}$ is a transient Markov chain.

Pitman also introduced parametrized extensions of this process. Of these, the processes with an infinite base set are $h$-transforms of each other, and the Doob-Martin compactification can be given explicitly [5]. The elements of the boundary may be represented by probability distributions $\rho=\left(\rho_{k}\right)_{k \in \mathbb{N}}$ on $\mathbb{N}$, and, conditionally on the limit being $\rho$, the random sequence $\left(K_{n}\right)_{n \in \mathbb{N}}$ of partition sizes (number of occupied tables in the restaurant model) is identical in 
distribution to $\left(Y_{n}+1\right)_{n \in \mathbb{N}}$, where $Y_{n}$ is the rightmost value in the pruned sample sequence associated with the distribution $\tilde{\rho}=\left(\tilde{\rho}_{k}\right)_{k \in \mathbb{N}_{0}}$ on $\mathbb{N}_{0}$ given by $\tilde{\rho}_{k}:=\rho_{k+1}, k \in \mathbb{N}_{0}$. This is also related to Kingman's paint box construction; again, we refer the reader to [11] for details and further references.

We are interested in the asymptotic behaviour of $Y_{n}$, especially in the way the asymptotics depend on the behaviour of the tail probabilities defined in (2). We will see that, again, there are phase transitions when the tail behaviour moves from the thin to the heavy end of the spectrum.

In the next section we list and discuss our results; proofs are deferred to Section 3.

\section{Results}

We begin at the thin end, comparing the $Y_{n}$ s to the maxima

$$
M_{n}:=\max \left\{X_{1}, \ldots, X_{n}\right\}
$$

of the samples. We have $\mathbb{P}\left(M_{n}<k\right)=\left(1-q_{k}\right)^{n}$ for all $k, n \in \mathbb{N}$, i.e. the distribution of the $M$-variables depends on the distribution of the $X$-variables in a simple and explicit way.

Clearly, $Y_{n} \leq M_{n}$ for all $n \in \mathbb{N}$. Also, a jump in the $M$-sequence entails a jump of size 1 in the $Y$-sequence, but note that a jump in the $M$-sequence may be larger than 1, and that the $Y$-sequence may jump where the $M$-sequence does not. The following result shows that, for rapidly decreasing tails, we have, with probability $1, Y_{n}=M_{n}$ eventually.

Theorem 1. The sequence $\left(M_{n}-Y_{n}\right)_{n \in \mathbb{N}}$ converges to 0 with probability 1 as $n \rightarrow \infty$ if and only if

$$
\sum_{k=0}^{\infty} \frac{q_{k+1}}{q_{k}}<\infty .
$$

We also have a sufficient condition for convergence in probability in terms of $\left(q_{k}\right)_{k \in \mathbb{N}}$. For a partial converse, i.e. in order to deduce this condition from the in-probability behaviour of the differences, we use a specific random subsequence of the full sequence.

Theorem 2. If

$$
\lim _{k \rightarrow \infty} \frac{q_{k+1}}{q_{k}}=0
$$

then the sequence $\left(M_{n}-Y_{n}\right)_{n \in \mathbb{N}}$ converges to 0 in probability as $n \rightarrow \infty$. Furthermore, with

$$
\tau_{m}:=\inf \left\{n \in \mathbb{N}: Y_{n}=m\right\}, \quad m \in \mathbb{N},
$$

convergence of the sequence $\left(M_{\tau_{m}}-Y_{\tau_{m}}\right)_{m \in \mathbb{N}}$ to 0 in probability as $m \rightarrow \infty$ implies (4).

We note in passing that Theorems 1 and 2 together imply that, for samples from a Poisson distribution, the differences $M_{n}-Y_{n}$ converge to 0 in probability but not almost surely.

As in [7], the geometric case turns out to be of special interest, in view of its appearance in connection with the DST algorithm, and also in view of its role as a borderline case. Because of our general assumption (1), we use the 'number of failures' version $\operatorname{Geo}_{0}(p)$; see also Remark 1 of [7]. We also put $r:=1 / q$. It is well known that, asymptotically, the sequence of maxima for samples from this distribution, after an appropriate shift, exhibits periodic distributional fluctuations. Indeed, with a suitable background construction, see, e.g. [6, p. 454], we see that $M_{n_{m}}-\left\lfloor\log _{r} m_{n}\right\rfloor$ converges in distribution along subsequences $\left(n_{m}\right)_{m \in \mathbb{N}}$ that are such that

$$
\lim _{m \rightarrow \infty}\left\{\log _{r} n_{m}\right\}=\eta \quad \text { for some } \eta \in[0,1) .
$$


Here we have written $\lfloor x\rfloor:=\max \{k \in \mathbb{Z}: k \leq x\}$ for the 'floor' of $x$ and $\{x\}:=x-\lfloor x\rfloor$ for its fractional part. The limit distribution is the same as the distribution of $\left\lfloor(\log r) Z_{1}+\eta\right\rfloor$, with $\mathbb{P}\left(Z_{1} \leq z\right)=\mathrm{e}^{-\mathrm{e}^{-z}}$, the Gumbel distribution. A similar result holds for $\left(Y_{n}\right)_{n \in \mathbb{N}}$; see [3]. Along subsequences $\left(n_{m}\right)_{m \in \mathbb{N}}$ that satisfy (5), the sequence $\left(Y_{n_{m}}-\left\lfloor\log _{r} m_{n}\right\rfloor\right)_{m \in \mathbb{N}}$ converges in distribution, where the distribution of the limit is the same as the distribution of $\left\lfloor(-\log r) Z_{2}+\eta\right\rfloor$, with $Z_{2}:=\sum_{j=1}^{\infty} q^{j} V_{j}$ and $\left(V_{j}\right)_{j \in \mathbb{N}}$ an independent and identically distributed sequence of standard exponentials (Dennert and Grübel [3] only treated the case $p=\frac{1}{2}$, but the extension to general $p$ is straightforward). Remarkably, for the differences, the periodicities cancel.

Theorem 3. If the random variables $X_{i}$ are independent and $X_{i} \sim \mathrm{Geo}_{0}(p)$ for all $i \in \mathbb{N}$, then

$$
\lim _{n \rightarrow \infty} \mathbb{P}\left(M_{n}-Y_{n}=k\right)=\pi_{k}:=\frac{a_{k}}{\sum_{l=0}^{\infty} a_{l}} \quad \text { for all } k \in \mathbb{N}_{0},
$$

where the sequence $\left(a_{k}\right)_{k \in \mathbb{N}_{0}}$ is given recursively by $a_{0}:=1$ and

$$
a_{k+1}:=\frac{1}{1-q^{k+1}}\left(q a_{k}-p \sum_{j=0}^{k-1} q^{k-j} a_{j}\right) \quad \text { for all } k \in \mathbb{N}_{0} .
$$

In the proof we will see that $a_{k}>0$ for all $k \in \mathbb{N}$, and that indeed $\sum_{l=0}^{\infty} a_{l}<\infty$.

At the heavy end of the spectrum of tail behaviour, $M_{n}$ and $Y_{n}$ drift apart as $n \rightarrow \infty$; hence, we deal with the $Y_{n}$ s directly. The following result on sums of geometric random variables is our main tool for this range of tail behaviour; we believe that it is of interest in its own right.

Theorem 4. Assume that the random variables $G_{k}, k \in \mathbb{N}$, are independent and that $G_{k} \sim \operatorname{Geo}_{1}\left(q_{k}\right)$, with $q_{k}=\mathbb{P}\left(X_{1} \geq k\right)$ as in (2). Set $S_{n}=\sum_{k=1}^{n} G_{k}$. Assume further that

$$
\frac{q_{k+1}}{q_{k}} \rightarrow 1 \text { as } n \rightarrow \infty .
$$

(a) Then

$$
\frac{S_{n}}{\mathbb{E} S_{n}} \stackrel{\mathbb{P}}{\rightarrow} 1 \text { as } n \rightarrow \infty .
$$

(b) For all $m \in \mathbb{N}$, let

$$
r_{m}:=\frac{\mathbb{E} S_{m}}{\mathbb{E} G_{m}}=q_{m} \sum_{k=1}^{m} \frac{1}{q_{k}} .
$$

Then

$$
\frac{S_{n}}{\mathbb{E} S_{n}} \stackrel{\text { a.s. }}{\longrightarrow} 1 \text { as } n \rightarrow \infty
$$

if and only if the following condition holds:

$$
\sum_{m=1}^{\infty} \mathrm{e}^{-\delta r_{m}}<\infty \text { for all } \delta>0
$$

In Section 3.5 we will show that (6) implies that $r_{m} \rightarrow \infty$, but that the rate may be arbitrarily slow. In particular, condition (7) is not vacuous. 
In order to use this for the pruned samples, we first recall that a function $L: \mathbb{R}_{+} \rightarrow \mathbb{R}$ is said to be slowly varying (at $\infty$ ) if

$$
\lim _{x \rightarrow \infty} \frac{L(\alpha x)}{L(x)}=1 \quad \text { for all } \alpha>0 .
$$

A standard example is $L(x)=(\log x)^{\beta}, \beta \in \mathbb{R}$. Clearly, if $L$ is slowly varying then so is $x \mapsto 1 / L(x)$. Such a function $L$ has a de Bruijn conjugate $L^{\#}$, unique up to asymptotic equivalence, which is again slowly varying, and satisfies

$$
\lim _{x \rightarrow \infty} L(x) L^{\#}(x L(x))=1 \quad \text { and } \quad \lim _{x \rightarrow \infty} L^{\#}(x) L\left(x L^{\#}(x)\right)=1 .
$$

This is Theorem 1.5.13 of [1], our basic reference for this area. For $L(x)=(\log x)^{\beta}$, we obtain $L^{\#}=1 / L$, i.e. $L^{\#}(x)=(\log x)^{-\beta}$.

Note that $\left(Y_{n}\right)_{n \in \mathbb{N}}$ is the renewal process associated with the lifetimes $\left(G_{k}\right)_{k \in \mathbb{N}}$ from Theorem 4. We now obtain our main result for the heavy-tailed case by a process known as renewal inversion. We write $a_{n} \sim b_{n}$ for two real sequences $\left(a_{n}\right)_{n \in \mathbb{N}}$ and $\left(b_{n}\right)_{n \in \mathbb{N}}$ if $\lim _{n \rightarrow \infty}\left(a_{n} / b_{n}\right)=1$.

Theorem 5. Suppose that, for some $\alpha>0$,

$$
q_{k} \sim k^{-\alpha} L(k) \text { as } k \rightarrow \infty,
$$

with L slowly varying. Then, with probability 1 ,

$$
Y_{n} \sim n^{1 /(1+\alpha)} L_{0}(n) \text { as } n \rightarrow \infty,
$$

where $L_{0}$ is the de Bruijn conjugate of $1 / L$.

\section{Proofs}

For very thin tails, we use a pathwise argument that builds on the corresponding result in [7]. For moderately thin tails, we use suitable bounds for the probability in question, together with direct arguments. The geometric case is approached via a suitable bivariate Markov chain. For heavy tails, we first prove the result on sums of geometrically distributed random variables and then obtain the result on the asymptotics of pruned samples.

In addition to the processes $X=\left(X_{n}\right)_{n \in \mathbb{N}}, Y=\left(Y_{n}\right)_{n \in \mathbb{N}}$, and $M=\left(M_{n}\right)_{n \in \mathbb{N}}$, we define another process $\Delta=\left(\Delta_{n}\right)_{n \in \mathbb{N}}$ by $\Delta_{n}:=M_{n}-Y_{n}, n \in \mathbb{N}$.

\subsection{Proof of Theorem 1}

Suppose first that (3) is satisfied. Let $v_{1}:=1$ and, for $k>1$,

$$
v_{k}:=\inf \left\{n>v_{k-1}: X_{n}>X_{v_{k-1}}\right\}
$$

be the jump times of the process $M$. With probability 1 , these are all finite. Let

$$
A_{k}:=\left\{\omega \in \Omega: X_{n}(\omega)=M_{v_{k}}(\omega) \text { for some } n \in\left\{v_{k}(\omega)+1, \ldots, v_{k+1}(\omega)-1\right\}\right\}
$$

be the event that the $X$-process visits the old maximum before it achieves the next new maximum. Let $A_{\infty}:=\limsup _{k \rightarrow \infty} A_{k}$ be the event that infinitely many of the $A_{k} \mathrm{~s}, k \in \mathbb{N}$, happen. Finally, let

$$
B_{\infty}:=\left\{\omega \in \Omega: \text { there exists a } k_{0} \text { such that, for all } k \geq k_{0}, M_{v_{k+1}}(\omega)=M_{v_{k}}(\omega)+1\right\}
$$

be the event that, eventually, the jumps of $M$ all have size 1. 
Let $\omega \in A_{\infty} \cap B_{\infty}$, with $k_{0}=k_{0}(\omega)$ as in (9). Suppose that $\Delta_{v_{k_{0}}}(\omega)=j$. Consider the evolution of the associated $M$ - and $Y$-paths from $n=v_{k_{0}}(\omega)$ onwards: $M$ jumps by at most 1 , and then $Y$ also jumps by 1; furthermore, if $Y$ visits the old maximum between two jump times, then $\Delta$ decreases by 1 if it is greater than 0 . By the definition of $A_{\infty}$, there will be infinitely many of these visits; hence, $\Delta_{n}(\omega)=0$ from some $n_{0}=n_{0}(\omega)$ onwards.

For the proof of sufficiency of (3), it is therefore enough to show that $\mathbb{P}\left(B_{\infty}\right)=1$ and $\mathbb{P}\left(A_{\infty}\right)=1$. The first of these is an immediate consequence of Theorem 1 of [7]. For the second, we consider $A_{k}$ : on $M_{v_{k}}=j$, this is the event that the post- $v_{k}$ process $\left(X_{v_{k}+n}\right)_{n \in \mathbb{N}}$ hits $j$ when it first leaves the set $\{0, \ldots, j-1\}$. A straightforward calculation leads to

$$
\mathbb{P}\left(A_{k} \mid M_{v_{k}}=j\right)=\frac{p_{j}}{q_{j}} .
$$

From (1) and (3), it follows that

$$
\alpha:=\inf _{j \in \mathbb{N}_{0}} \frac{p_{j}}{q_{j}}>0 .
$$

The post- $v_{k}$ process $\left(X_{v_{k}+n}\right)_{n \in \mathbb{N}}$ and the pre- $v_{k}$ process $\left(X_{v_{k} \wedge n}\right)_{n \in \mathbb{N}}$ are independent; hence, (10) and (11) together lead to

$$
\mathbb{P}\left(A_{k} \mid \mathcal{F}_{\nu_{k}}\right) \geq \alpha \quad \text { for all } k \in \mathbb{N},
$$

with $\left(\mathcal{F}_{n}\right)_{n \in \mathbb{N}}$ the natural filtration associated with the process $X$. Clearly, $A_{k} \in \mathcal{F}_{v_{k+1}}$, and $\mathbb{P}\left(A_{\infty}\right)=1$ now follows with Lévy's generalization of the Borel-Cantelli lemma; see, e.g. [8, Corollary 6.20].

For the proof of the necessity of (3), we note that, as $\Delta_{n}$ has nonnegative integer values, if $\Delta_{n} \rightarrow 0$ almost surely then there is a set $A$ with $\mathbb{P}(A)=1$ such that,

$$
\text { for all } \omega \in A \text {, there exists an } n_{0} \in \mathbb{N} \text { such that, for all } n \geq n_{0}, \Delta_{n}(\omega)=0 .
$$

On this set, the gaps will eventually vanish as the $Y$-process can only increase by steps of size 1; hence, we can apply the other direction of Theorem 1 of [7].

\subsection{Proof of Theorem 2}

We first show that condition (4) is sufficient. For this, we need the following auxiliary statement.

Lemma 1. If $q_{k+1} / q_{k} \rightarrow 0$ as $k \rightarrow \infty$ then

$$
q_{m} \sum_{j=1}^{m-1} \frac{1}{q_{j}} \rightarrow 0 \quad \text { as } m \rightarrow \infty
$$

Proof. Let $\varepsilon>0$ be given. For $\eta>0$, pick $k_{0} \in \mathbb{N}$ such that $q_{k+1} / q_{k}<\eta$ for $k \geq k_{0}$. Then, for $m>k_{0}$, we have

$$
q_{m} \sum_{j=1}^{m-1} \frac{1}{q_{j}}=q_{m} \sum_{j=1}^{k_{0}-1} \frac{1}{q_{j}}+\sum_{j=k_{0}}^{m-1} \frac{q_{m}}{q_{j}}<q_{m} \sum_{j=1}^{k_{0}-1} \frac{1}{q_{j}}+\sum_{j=k_{0}}^{m-1} \eta^{m-j}<q_{m} \sum_{j=1}^{k_{0}-1} \frac{1}{q_{j}}+\frac{\eta}{1-\eta} .
$$

If $\eta$ is such that $\eta<\min \left\{\varepsilon / 3, \frac{1}{2}\right\}$ then $\eta /(1-\eta)<2 \eta<2 \varepsilon / 3$. Since $q_{m} \rightarrow 0$, we can find an $m_{0}$ such that, for $m \geq m_{0}, q_{m} \sum_{j=1}^{k_{0}-1} 1 / q_{j}<\varepsilon / 3$. This proves the lemma. 
We next show that (4) implies that $M_{n}-Y_{n} \rightarrow 0$ in probability, which is the first part of Theorem 2 .

Take $\varepsilon>0$, and consider $\mathbb{P}\left(M_{n}-Y_{n}>\varepsilon\right)$. Since both random variables are integer valued we have, for all $m_{0} \in \mathbb{N}$,

$$
\begin{aligned}
\mathbb{P}\left(M_{n}-Y_{n}>\varepsilon\right) & \leq \mathbb{P}\left(M_{n} \geq Y_{n}+1\right) \\
& =\sum_{m=1}^{\infty} \mathbb{P}\left(M_{n} \geq m, Y_{n}=m-1\right) \\
& \leq \mathbb{P}\left(Y_{n} \leq m_{0}\right)+\sum_{m=m_{0}}^{\infty} \mathbb{P}\left(M_{n} \geq m, Y_{n}=m-1\right) \\
& =\mathbb{P}\left(Y_{n} \leq m_{0}\right)+\sum_{m=m_{0}}^{\infty} \mathbb{P}\left(M_{n} \geq m \mid Y_{n}=m-1\right) \mathbb{P}\left(Y_{n}=m-1\right) .
\end{aligned}
$$

The key step is the following bound on the conditional probability.

Lemma 2. For any $m \geq 1$, we have

$$
\mathbb{P}\left(M_{n} \geq m \mid Y_{n}=m-1\right)=1-\prod_{j=1}^{m-1}\left(1-\frac{q_{m}}{q_{j}}\right) \leq q_{m} \sum_{j=1}^{m-1} \frac{1}{q_{j}} .
$$

Once we prove this lemma the proof of the theorem may be completed as follows. For $\eta>0$, first pick $m_{0} \in \mathbb{N}$ such that, for $m \geq m_{0}, q_{m} \sum_{j=1}^{m-1} 1 / q_{j}<\eta / 2$. This is possible by Lemma 1. Then, since $Y_{n} \rightarrow \infty$ in probability, there exists $n_{0} \in \mathbb{N}$ such that, for $n \geq n_{0}$, we have $\mathbb{P}\left(Y_{n} \leq m_{0}\right)<\eta / 2$. It now follows that, for $n \geq n_{0}$, we have

$$
\mathbb{P}\left(M_{n}-Y_{n}>\varepsilon\right) \leq \mathbb{P}\left(Y_{n} \leq m_{0}\right)+\frac{\eta}{2} \sum_{m=m_{0}}^{\infty} \mathbb{P}\left(Y_{n}=m-1\right) \leq \eta .
$$

Proof of Lemma 2. Describe the event $\left\{Y_{n}=m-1\right\}$ in terms of the sequence $\left(X_{k}\right)$. First, with $\tau_{0}:=0$ and $\tau_{m}(m \in \mathbb{N})$ as in the theorem,

$$
\left\{Y_{n}=m-1\right\}=\left\{\tau_{m-1} \leq n<\tau_{m}\right\}=\bigcup_{1 \leq k_{1}<\cdots<k_{m-1} \leq n} \bigcap_{j=1}^{m-1}\left\{\tau_{j}=k_{j}\right\} \cap\left\{\tau_{m}>n\right\} .
$$

Note that the union is over pairwise disjoint events. Therefore, it is enough to show that, for all $1 \leq k_{1}<\cdots<k_{m-1} \leq n$ and $m \geq 1$,

$$
\mathbb{P}\left(M_{n} \geq m \mid \bigcap_{j=1}^{m-1}\left\{\tau_{j}=k_{j}\right\} \cap\left\{\tau_{m}>n\right\}\right)=1-\prod_{j=1}^{m-1}\left(1-\frac{q_{m}}{q_{j}}\right) .
$$

Fix $1 \leq k_{1}<\cdots<k_{m-1} \leq n$. The event $\bigcap_{j=1}^{m-1}\left\{\tau_{j}=k_{j}\right\} \cap\left\{\tau_{m}>n\right\}$ means that

$$
\begin{aligned}
& X_{\left[1: k_{1}-1\right]}^{*}=0, X_{k_{1}} \geq 1, X_{\left[k_{1}+1, k_{2}-1\right]}^{*} \leq 1, X_{k_{2}} \geq 2, \ldots, \\
& X_{k_{m-1}} \geq m-1, X_{\left[k_{m-1}+1, n\right]}^{*} \leq m-1,
\end{aligned}
$$


where, for a set $A \subset \mathbb{N}, X_{A}^{*}=\max _{k \in A} X_{k}$, and, for nonnegative integers $a \leq b$, $[a, b]=\{a, a+1, \ldots, b\}$. Therefore, conditionally on this event, $M_{n} \geq m$ means that $\max _{1 \leq j \leq m-1} X_{k_{j}} \geq m$. Thus, the conditional probability in (13) is

$$
\begin{aligned}
& \mathbb{P}\left(\max _{1 \leq j<m} X_{k_{j}} \geq m \mid \bigcap_{j=1}^{m-1}\left\{\tau_{j}=k_{j}\right\} \cap\left\{\tau_{m}>n\right\}\right) \\
& \quad=1-\mathbb{P}\left(\bigcap_{j=1}^{m-1}\left\{X_{k_{j}}<m\right\} \mid \bigcap_{j=1}^{m-1}\left\{\tau_{j}=k_{j}\right\} \cap\left\{\tau_{m}>n\right\}\right) .
\end{aligned}
$$

By the above description in terms of random variables $\left(X_{k}\right)$ of the event we are conditioning on, the independence of the $X_{k} \mathrm{~s}$, and the definition of the conditional probability, we see that the last probability is

$$
\frac{\mathbb{P}\left(j \leq X_{k_{j}}<m ; j=1, \ldots, m-1\right)}{\mathbb{P}\left(X_{k_{j}} \geq j ; j=1, \ldots, m-1\right)}=\prod_{j=1}^{m-1} \frac{q_{j}-q_{m}}{q_{j}}=\prod_{j=1}^{m-1}\left(1-\frac{q_{m}}{q_{j}}\right) .
$$

This shows (13) and completes the proof as the inequality in (12) is clear.

For the proof of the second part of Theorem 2, we begin with

$$
\mathbb{P}\left(X_{\tau_{m}}=j\right)=\sum_{l=1}^{\infty} \mathbb{P}\left(X_{l}=j \mid \tau_{m}=l\right) \mathbb{P}\left(\tau_{m}=l\right) .
$$

In view of $\left\{\tau_{m}=l\right\}=\left\{X_{l} \geq m\right\} \cap\left\{Y_{l-1}=m-1\right\}$ and the fact that $Y_{l-1}$ is a function of $X_{1}, \ldots, X_{l-1}$ we have $\mathbb{P}\left(X_{l}=j \mid \tau_{m}=l\right)=\mathbb{P}\left(X_{1}=j \mid X_{1} \geq m\right)$, which leads to

$$
\mathbb{P}\left(\Delta_{\tau_{m}}>0\right) \geq \mathbb{P}\left(X_{\tau_{m}} \geq m+1\right)=\mathbb{P}\left(X_{1} \geq m+1 \mid X_{1} \geq m\right)=\frac{q_{m+1}}{q_{m}} .
$$

Hence, if (4) does not hold, i.e. if $\limsup _{m \rightarrow \infty}\left(q_{m+1} / q_{m}\right)>0$, then $\mathbb{P}\left(\Delta_{\tau_{m}}>0\right)$ cannot converge to 0 .

\subsection{Proof of Theorem 3}

We require the following auxiliary result which roughly says that the limit distribution of a process does not change if its clock is slowed down in a specific manner.

Proposition 1. Suppose that $Y=\left(Y_{n}\right)_{n \in \mathbb{N}}$ and $D=\left(D_{n}\right)_{n \in \mathbb{N}_{0}}$ are independent Markov chains, where $Y$ is of pure birth type with birth probabilities

$$
\mathbb{P}\left(Y_{n+1}=i+1 \mid Y_{n}=i\right)=1-\mathbb{P}\left(Y_{n+1}=i \mid Y_{n}=i\right)=\mu_{i}>0 \text { for all } i \in \mathbb{N}_{0},
$$

and start at 0 , and that $D$ is irreducible and aperiodic, with state space $E$, transition probabilities

$$
\mathbb{P}\left(D_{n+1}=j \mid D_{n}=i\right)=p_{D}(i, j) \quad \text { for all } i, j \in E,
$$

and stationary distribution $\pi=\left(\pi_{i}\right)_{i \in E}$. Let $Z=\left(Z_{n}\right)_{n \in \mathbb{N}}$ be defined by

$$
Z_{n}:=\left(Y_{n}, D_{Y_{n}}\right) \text { for all } n \in \mathbb{N} \text {. }
$$


Then $Z$ is a Markov chain with state space $\mathbb{N}_{0} \times E$ and transition probabilities

$$
\begin{gathered}
p_{Z}((i, j),(i, j))=1-\mu_{i} \quad \text { for all } i \in \mathbb{N}_{0}, j \in E, \\
p_{Z}((i, j),(i+1, k))=\mu_{i} p_{D}(j, k) \quad \text { for all } i \in \mathbb{N}_{0}, j, k \in E .
\end{gathered}
$$

Furthermore,

$$
\lim _{n \rightarrow \infty} \mathbb{P}\left(D_{Y_{n}}=k\right)=\pi_{k} \quad \text { for all } k \in E .
$$

Proof. We first show that

$$
\mathbb{P}\left(Z_{n+1}=\left(i_{n+1}, j_{n+1}\right) \mid Z_{0}=\left(i_{0}, j_{0}\right), \ldots, Z_{n}=\left(i_{n}, j_{n}\right)\right)
$$

is the same as

$$
\mathbb{P}\left(Z_{n+1}=\left(i_{n+1}, j_{n+1}\right) \mid Z_{n}=\left(i_{n}, j_{n}\right)\right) .
$$

For this, it is enough to consider paths with $i_{n+1} \in\left\{i_{n}, i_{n}+1\right\}$ and $j_{n+1}=j_{n}$ if $i_{n+1}=i_{n}$. Also, $\left(i_{0}, \ldots, i_{n}\right)$ has to be of the form

$$
(\underbrace{0, \ldots, 0}_{\nu_{0}}, \underbrace{1, \ldots, 1}_{v_{1}}, \ldots, \underbrace{l, \ldots, l}_{\nu_{l}})
$$

with $l \in \mathbb{N}_{0}$ and $v_{0}+\cdots+v_{l}=n$. Then

$$
\left(j_{0}, \ldots, j_{n}\right)=(\underbrace{k_{0}, \ldots, k_{0}}_{\nu_{0}}, \underbrace{k_{1}, \ldots, k_{1}}_{\nu_{1}}, \ldots, \underbrace{k_{l}, \ldots, k_{l}}_{\nu_{l}}) .
$$

In such cases (17) reduces to either

$$
\mathbb{P}\left(Y_{n+1}=l, D_{l}=k_{l} \mid Y_{0}=0, \ldots, Y_{n}=l, D_{0}=k_{0}, \ldots, D_{l}=k_{l}\right)
$$

or

$$
\mathbb{P}\left(Y_{n+1}=l+1, D_{l+1}=k_{l+1} \mid Y_{0}=0, \ldots, Y_{n}=l, D_{0}=k_{0}, \ldots, D_{l}=k_{l}\right),
$$

which equals $1-\mu_{l}$ or $\mu_{l} p_{D}\left(k_{l} \cdot k_{l+1}\right)$, respectively. The same values appear for (18). This shows that $Z$ is a Markov chain and also confirms (14) and (15).

For the proof of (16), let $k \in E$ and $\varepsilon>0$. A decomposition with respect to the value of the clock together with the independence of $Y$ and $D$ results in

$$
\mathbb{P}\left(D_{Y_{n}}=k\right)=\sum_{m=0}^{\infty} \mathbb{P}\left(D_{m}=k\right) \mathbb{P}\left(Y_{n}=m\right) .
$$

The ergodic theorem for Markov chains gives $\lim _{m \rightarrow \infty} \mathbb{P}\left(D_{m}=k\right)=\pi_{k}$, so, for a suitable $m_{0}$,

$$
\left|\mathbb{P}\left(D_{m}=k\right)-\pi_{k}\right| \leq \frac{1}{2} \varepsilon \quad \text { for all } m \geq m_{0} .
$$

As the birth probabilities are all strictly greater than 0 , it follows further that $Y_{n}$ converges to $\infty$ with probability 1 , and, hence, in probability. This implies that, for some $n_{0}$,

$$
\mathbb{P}\left(Y_{n}<m_{0}\right) \leq \frac{1}{2} \varepsilon \quad \text { for all } n \geq n_{0} .
$$


Combining these we obtain, for all $n \geq n_{0}$,

$$
\begin{aligned}
\left|\mathbb{P}\left(D_{Y_{n}}=k\right)-\pi_{k}\right| & \leq\left(\sum_{m=0}^{m_{0}-1}+\sum_{m=m_{0}}^{\infty}\right)\left|\mathbb{P}\left(D_{m}=k\right)-\pi_{k}\right| \mathbb{P}\left(Y_{n}=m\right) \\
& \leq \mathbb{P}\left(Y_{n}<m_{0}\right)+\sup _{m \geq m_{0}}\left|\mathbb{P}\left(D_{m}=k\right)-\pi_{k}\right| \\
& \leq \varepsilon .
\end{aligned}
$$

This completes the proof of the proposition.

We remark in passing that, in contrast to $Y$ and $D$, the process $\left(D_{Y_{n}}\right)_{n \in \mathbb{N}}$ is not a timehomogeneous Markov chain; indeed, if $\lim _{i \rightarrow \infty} \mu_{i}=0$ then the 'stay where you are' probabilities $\mathbb{P}\left(D_{Y_{n+1}}=k \mid D_{Y_{n}}=k\right)$ tend to 1 as $n \rightarrow \infty$ for each $k \in E$.

For the proof of Theorem 3, we now define the bivariate process $V=\left(V_{n}\right)_{n \in \mathbb{N}}$ by $V_{n}:=$ $\left(Y_{n}, \Delta_{n}\right)$ for all $n \in \mathbb{N}$. The dynamics of $V$ are given by $V_{n+1}=\Phi\left(V_{n}, X_{n+1}\right)$ with

$$
\Phi((i, j), k)= \begin{cases}(i, j) & \text { if } k \leq i, \\ (i+1, j-1) & \text { if } i<k \leq i+j, \\ (i+1, j+l-1) & \text { if } k=i+j+l \text { for some } l \in \mathbb{N} .\end{cases}
$$

This implies that $V$ is a Markov chain with state space $\mathbb{N}_{0} \times \mathbb{N}_{0}$ and transition probabilities

$$
\begin{gathered}
p_{V}((i, j),(i, j))=1-q_{i+1}, \\
p_{V}((i, j),(i+1, j-1))=q_{i+1}-q_{i+j+1}, \\
p_{V}((i, j),(i+1, j+k))=p_{i+j+k+1}, \quad k \in \mathbb{N}_{0},
\end{gathered}
$$

in the general case. For $\operatorname{Geo}_{0}(p)$, as in the theorem, we obtain

$$
\begin{gathered}
p_{V}((i, j),(i, j))=1-q^{i+1}, \\
p_{V}((i, j),(i+1, j-1))=q^{i+1}\left(1-q^{j}\right), \\
p_{V}((i, j),(i+1, j+k))=q^{i+1} p q^{j+k}, \quad k \in \mathbb{N}_{0} .
\end{gathered}
$$

Now let $D=\left(D_{n}\right)_{n \in \mathbb{N}_{0}}$ be a Markov chain, independent of $Y$, with state space $E=\mathbb{N}_{0}$, starting at 0 , and transitions

$$
p_{D}(j, j-1)=1-q^{j}, \quad p_{D}(j, j+k)=p q^{j+k} \quad \text { for all } k \in \mathbb{N}_{0} .
$$

Clearly, $D$ is irreducible and aperiodic, and positive recurrence follows with Foster's theorem [2, p. 167].

Let $Z=\left(Z_{n}\right)_{n \in \mathbb{N}}$ with $Z_{n}:=\left(Y_{n}, D_{Y_{n}}\right)$ for all $n \in \mathbb{N}$. By Proposition $1, Z$ is a Markov chain and, on the basis of the above computations, it is easily checked that $p_{Z}=p_{V}$. Markov chains with the same transition probabilities and the same initial value are equal in distribution; in particular, $\left(\Delta_{n}\right)_{n \in \mathbb{N}}$ has the same distribution as $\left(D_{Y_{n}}\right)_{n \in \mathbb{N}}$. The final statement in Proposition 1 now implies that $\Delta_{n}$ converges in distribution to the stationary distribution $\pi$ of $D$. Invariance of $\pi$ under the transition mechanism (19) means that $\pi$ solves

$$
\pi_{k}=\left(1-q^{k+1}\right) \pi_{k+1}+\sum_{j=0}^{k} p q^{j} \pi_{k-j} \quad \text { for all } k \in \mathbb{N}_{0},
$$

which implies that it is of the form given in the theorem. 


\subsection{Proof of Theorem 4}

We begin with a lemma.

Lemma 3. Assume that $q_{k+1} / q_{k} \rightarrow 1$ as $k \rightarrow \infty$. Then, as $m \rightarrow \infty$,

$$
q_{m} \sum_{k=1}^{m} \frac{1}{q_{k}} \rightarrow \infty
$$

but it may go to $\infty$ at an arbitrarily slow rate.

Proof. For the first assertion, take an arbitrary $M>0$. Since $q_{k+1} / q_{k} \rightarrow 1$, there exists an $m_{0}$ such that, for $m \geq m_{0}$, we have $q_{m+1} / q_{m}>1-1 / M$. Then, for $m>m_{0}$,

$$
\begin{aligned}
q_{m} \sum_{k=1}^{m} \frac{1}{q_{k}} & \geq \sum_{k=m_{0}+1}^{m} \frac{q_{m}}{q_{k}} \\
& \geq \sum_{k=m_{0}+1}^{m}\left(1-\frac{1}{M}\right)^{m-k} \\
& =\sum_{j=0}^{m-m_{0}-1}\left(1-\frac{1}{M}\right)^{j} \\
& =M\left(1-\left(1-\frac{1}{M}\right)^{m-m_{0}}\right) \\
& \geq M\left(1-\mathrm{e}^{-1}\right),
\end{aligned}
$$

provided $m-m_{0} \geq M$. This shows (20).

To prove the second assertion of the lemma, let $k_{1}, k_{2}, \ldots \in \mathbb{N}$ and $\varepsilon_{1}, \varepsilon_{2}, \ldots$ decreasing to 0 be given. Let the first $k_{1} q_{m}$ s be defined by

$$
\left(1-\varepsilon_{1}\right),\left(1-\varepsilon_{1}\right)^{2}, \ldots,\left(1-\varepsilon_{1}\right)^{k_{1}},
$$

the next $k_{2}$ by

$$
\left(1-\varepsilon_{1}\right)^{k_{1}}\left(1-\varepsilon_{2}\right),\left(1-\varepsilon_{1}\right)^{k_{1}}\left(1-\varepsilon_{2}\right)^{2}, \ldots,\left(1-\varepsilon_{1}\right)^{k_{1}}\left(1-\varepsilon_{2}\right)^{k_{2}},
$$

and so on, with the last group of $k_{m} q_{k}$ s given by

$$
\prod_{j=1}^{m-1}\left(1-\varepsilon_{j}\right)^{k_{j}}\left(1-\varepsilon_{m}\right), \prod_{j=1}^{m-1}\left(1-\varepsilon_{j}\right)^{k_{j}}\left(1-\varepsilon_{m}\right)^{2}, \ldots, \prod_{j=1}^{m-1}\left(1-\varepsilon_{j}\right)^{k_{j}}\left(1-\varepsilon_{m}\right)^{k_{m}} .
$$

Set $K_{r}=\sum_{i=1}^{r} k_{i}$. Then, for $K_{r-1} \leq k \leq K_{r}$, we have

$$
\prod_{j=1}^{r}\left(1-\varepsilon_{j}\right)^{k_{j}} \leq q_{k} \leq \prod_{j=1}^{r-1}\left(1-\varepsilon_{j}\right)^{k_{j}}
$$

Hence,

$$
\sum_{k=K_{r-1}+1}^{K_{r}} \frac{1}{q_{k}} \leq k_{r} \prod_{j=1}^{r}\left(1-\varepsilon_{j}\right)^{-k_{j}}
$$


and, therefore, provided that the $k_{j}$ s are increasing,

$$
\sum_{k=1}^{K_{m}} \frac{1}{q_{k}} \leq k_{m} \sum_{k=1}^{m} \prod_{j=1}^{k}\left(1-\varepsilon_{j}\right)^{-k_{j}}
$$

It follows that, for $K_{m-1} \leq n \leq K_{m}$,

$$
\begin{aligned}
q_{n} \sum_{k=1}^{n} \frac{1}{q_{k}} & \leq k_{m} \prod_{j=1}^{m-1}\left(1-\varepsilon_{j}\right)^{k_{j}} \sum_{k=1}^{m} \prod_{j=1}^{k}\left(1-\varepsilon_{j}\right)^{-k_{j}} \\
& =k_{m} \sum_{k=1}^{m} \prod_{j=1}^{k}\left(1-\varepsilon_{j}\right)^{-k_{j}} \prod_{j=1}^{m-1}\left(1-\varepsilon_{j}\right)^{k_{j}} \\
& =k_{m}\left\{\sum_{k=1}^{m-1} \prod_{j=k+1}^{m-1}\left(1-\varepsilon_{j}\right)^{k_{j}}+\left(1-\varepsilon_{m}\right)^{-k_{m}}\right\},
\end{aligned}
$$

where, as usual, we adopt the convention that a product over an empty range is 1 . Now, upon setting $\varepsilon_{i}=1 / k_{i}$ we see that the term in the curly braces is bounded by an absolute constant $C \leq \mathrm{e} /(\mathrm{e}-1)+4$ (this may be seen by choosing $k_{1} \geq 2$ and using the elementary inequality $1-x \leq \mathrm{e}^{-x}$ to bound the first sum). Thus,

$$
q_{n} \sum_{k=1}^{n} \frac{1}{q_{k}} \leq C k_{m}
$$

which can go to $\infty$ arbitrarily slowly. This proves the lemma.

We now turn to the proof of Theorem 4 .

Recall that if $G \sim \mathrm{Geo}_{1}(p)$ then its moment generating function is

$$
\mathbb{E} \mathrm{e}^{\lambda G}=\frac{p \mathrm{e}^{\lambda}}{1-(1-p) \mathrm{e}^{\lambda}}=\left(1-\frac{1-\mathrm{e}^{-\lambda}}{p}\right)^{-1},
$$

whenever $\mathrm{e}^{\lambda}(1-p)<1$, i.e. $\lambda<-\log (1-p)$. Therefore, for any $t>0$, we have

$$
\mathbb{P}\left(S_{n}>t\right)=\mathbb{P}\left(\exp \left(\lambda S_{n}\right)>\mathrm{e}^{\lambda t}\right) \leq \mathrm{e}^{-\lambda t} \mathbb{E} \mathrm{e}^{\lambda S_{n}}=\mathrm{e}^{-\lambda t} \prod_{k=1}^{n}\left(1-\frac{1-\mathrm{e}^{-\lambda}}{q_{k}}\right)^{-1} .
$$

Note that, for any $\beta>1$, if $0 \leq x \leq(\beta-1) / \beta$ then $1 /(1-x) \leq \mathrm{e}^{\beta x}$. We will choose a suitable $\beta$ in a moment. For now, note that if $\lambda>0$ is such that $\left(1-\mathrm{e}^{-\lambda}\right) / q_{k}<(\beta-1) / \beta$ for each $k=1, \ldots, n$, we obtain

$$
\mathbb{P}\left(S_{n}>t\right) \leq \exp \left\{-\lambda t+\beta\left(1-\mathrm{e}^{-\lambda}\right) \sum_{k=1}^{n} \frac{1}{q_{k}}\right\} \leq \exp \left\{-\lambda\left(t-\beta \sum_{k=1}^{n} \frac{1}{q_{k}}\right)\right\}
$$

where in the last step we used the inequality $1-\mathrm{e}^{-\lambda} \leq \lambda$ valid for any $\lambda$. Now, fix arbitrary $\varepsilon>0$ and set $t=(1+\varepsilon) \sum_{k=1}^{n} 1 / q_{k}$. Then, choose $\beta>1$ so that $\delta:=1+\varepsilon-\beta>0$. 
Finally, set $\lambda=q_{n}(\beta-1) / \beta$. Then all the earlier requirements are satisfied (note that since $\left(q_{k}\right)$ is decreasing, we do have $1-\mathrm{e}^{-\lambda} \leq q_{k}(\beta-1) / \beta$ for every $\left.k \leq n\right)$ and we obtain

$$
\mathbb{P}\left(S_{n}>(1+\varepsilon) \mathbb{E} S_{n}\right) \leq \exp \left\{-\delta q_{n} \sum_{k=1}^{n} \frac{1}{q_{k}}\right\} .
$$

But this means that

$$
\mathbb{P}\left(\frac{S_{n}}{\mathbb{E} S_{n}}-1>\varepsilon\right) \leq \exp \left\{-\delta q_{n} \sum_{k=1}^{n} \frac{1}{q_{k}}\right\}
$$

To bound the lower tail

$$
\mathbb{P}\left(\frac{S_{n}}{\mathbb{E} S_{n}}-1<-\varepsilon\right),
$$

we proceed in a similar fashion: namely, for any $\lambda>0$, this last probability is equal to

$$
\begin{aligned}
\mathbb{P}\left(\mathrm{e}^{-\lambda S_{n}}>\mathrm{e}^{-\lambda(1-\varepsilon) E S_{n}}\right) & \leq \mathrm{e}^{\lambda(1-\varepsilon) E S_{n}} \prod_{k=1}^{n} \mathbb{E} \mathrm{e}^{-\lambda G_{k}} \\
& =\mathrm{e}^{\lambda(1-\varepsilon) E S_{n}} \prod_{k=1}^{n}\left(1+\frac{\mathrm{e}^{\lambda}-1}{q_{k}}\right)^{-1} .
\end{aligned}
$$

As before, we note that if $0<\beta<1$ then, for $0 \leq x \leq(1-\beta) / \beta$, we have $1 /(1+x) \leq \mathrm{e}^{-\beta x}$. Therefore, the right-hand side above is further bounded by

$$
\exp \left\{\lambda(1-\varepsilon) \mathbb{E} S_{n}-\beta\left(\mathrm{e}^{\lambda}-1\right) \sum_{k=1}^{n} \frac{1}{q_{k}}\right\} \leq \exp \left\{\lambda(1-\varepsilon-\beta) \sum_{k=1}^{n} \frac{1}{q_{k}}\right\}
$$

provided of course that, for a chosen $\beta, \lambda$ satisfies $\left(\mathrm{e}^{\lambda}-1\right) / q_{k} \leq(1-\beta) / \beta$ for every $1 \leq k \leq n$. It is clear that, for a given $\beta$, we may choose $\lambda=c q_{n}$ for a suitable constant $c$ dependent only on $\beta$. (In fact, we may choose $c=\log (1+(1-\beta) / \beta$ ). ) And now, as earlier, given $\varepsilon>0$, it is enough to choose $\beta<1$ so that $\delta:=\beta-(1-\varepsilon)>0$ to obtain

$$
\mathbb{P}\left(\frac{S_{n}}{\mathbb{E} S_{n}}-1<-\varepsilon\right) \leq \exp \left\{-\delta q_{n} \sum_{k=1}^{n} \frac{1}{q_{k}}\right\}
$$

Clearly, (21), (22), and (20) imply the first part and the sufficiency of (7) for the almost-sure convergence of $S_{n} / \mathbb{E} S_{n}$ in the second part of our theorem.

To prove the necessity of (7), suppose that $S_{n} / \mathbb{E} S_{n}$ converges almost surely. We will show that (7) holds (which in turn will imply that the limit has to be 1). Pick any $\delta>0$. Note that

$$
\frac{G_{n}}{\mathbb{E} S_{n}}=\frac{S_{n}}{\mathbb{E} S_{n}}-\frac{\mathbb{E} S_{n-1}}{\mathbb{E} S_{n}} \frac{S_{n-1}}{\mathbb{E} S_{n-1}}
$$

and that, by (20),

$$
\frac{\mathbb{E} S_{n-1}}{\mathbb{E} S_{n}}=1-\frac{\mathbb{E} G_{n}}{\mathbb{E} S_{n}}=1-\frac{1}{q_{n} \sum_{k=1}^{n} q_{k}^{-1}} \rightarrow 1
$$


It follows that $G_{n} / \mathbb{E} S_{n} \rightarrow 0$ almost surely. Take any $\delta_{1}$ such that $0<\delta_{1}<\delta$. Since the events $\left\{G_{m}>\delta_{1} \mathbb{E} S_{m}\right\}$ are independent, it follows by the Borel-Cantelli lemma that

$$
\sum_{m=1}^{\infty} \mathbb{P}\left(G_{m} \geq \delta_{1} \mathbb{E} S_{m}\right)<\infty .
$$

But, since $G_{m}$ is geometric with parameter $q_{m}$, we obtain

$$
\begin{aligned}
\mathbb{P}\left(G_{m} \geq \delta_{1} \mathbb{E} S_{m}\right) & \geq \mathbb{P}\left(G_{m} \geq\left\lceil\delta_{1} \mathbb{E} S_{n}\right\rceil\right) \\
& =\left(1-q_{m}\right)^{\left.\delta_{1} \mathbb{E} S_{m}\right\rceil-1} \\
& \geq\left(1-q_{m}\right)^{\delta_{1} \mathbb{E} S_{m}} \\
& =\left(\left(1-q_{m}\right)^{q_{m}^{-1} \delta_{1} \delta^{-1}}\right)^{\delta q_{m} \sum_{k=1}^{m} q_{k}^{-1}} .
\end{aligned}
$$

Since $\delta_{1} / \delta<1$, for all $m$ sufficiently large

$$
\left(1-q_{m}\right)^{q_{m}^{-1} \delta_{1} \delta^{-1}} \geq \mathrm{e}^{-1} .
$$

Thus,

$$
\sum_{m=1}^{\infty} \exp \left\{-\delta q_{m} \sum_{k=1}^{m} \frac{1}{q_{k}}\right\}<\infty
$$

which proves (7).

\subsection{Proof of Theorem 5}

From [1, Theorem 1.5.11] we obtain

$$
\mathbb{E} S_{m}=\sum_{k=1}^{m} \frac{1}{q_{k}} \sim \frac{m^{\alpha+1}}{L(m)} \quad \text { as } m \rightarrow \infty .
$$

Together with (8) this shows that (7) is satisfied. By Theorem 4 and (23),

$$
S_{m} \sim \frac{m^{\alpha+1}}{L(m)} \quad \text { as } m \rightarrow \infty,
$$

almost surely.

For a function $f: \mathbb{R}_{+} \rightarrow \mathbb{R}$, we define its 'inverse' $f \leftarrow$ by

$$
f^{\leftarrow}(y):=\inf \{x \in \mathbb{R}: f(x) \geq y\} .
$$

Then $n \rightarrow Y_{n}$ is the inverse of $m \rightarrow S_{m}$ in this sense, and the statement of the theorem now follows with [1, Proposition 1.5.15].

\section{References}

[1] Bingham, N. H., Goldie, C. M. And Teugels, J. L. (1987). Regular Variation (Encyclopedia Math. Appl. 27). Cambridge University Press.

[2] Brémaud, P. (1999). Markov Chains (Texts Appl. Math. 31). Springer, New York.

[3] Dennert, F. AND GrüBEL, R. (2007). Renewals for exponentially increasing lifetimes, with an application to digital search trees. Ann. Appl. Prob. 17, 676-687.

[4] Drmota, M. (2009). Random Trees. SpringerWienNewYork, Vienna. 
[5] Evans, S. N., Grübel, R. And Wakolbinger, A. (2012). Trickle-down processes and their boundaries. Electron J. Prob. 17, 58pp.

[6] GRÜBEL, R. (2007). Distributional asymptotics in the analysis of algorithms: periodicities and discretization. In AofA '07 Conf. on Analysis of Algorithms (Discrete Math. Theoret. Comput. Sci. Proc. AH), pp. 451-462.

[7] Grübel, R. and Hitczenko, P. (2009). Gaps in discrete random samples. J. Appl. Prob. 46, 1038-1051.

[8] Kallenberg, O. (1997). Foundations of Modern Probability. Springer, New York.

[9] Knuth, D. E. (1973). The Art of Computer Programming, Vol. 3. Addison-Wesley, Reading, MA.

[10] Mahmoud, H. M. (1992). Evolution of Random Search Trees. John Wiley, New York.

[11] Pitman, J. (2006). Combinatorial Stochastic Processes (Lecture Notes Math. 1875). Springer, Berlin. 